Research Article

\title{
Preparation of Microfiber Grating for Real-Time Sensing of Escherichia Coli Concentration
}

\author{
Qingyan Qiu, Min Dai (iD, Caidong Luo, Ying Mu, Wei Du, Yu Li, Jun Luo, and Rong Li \\ Mianyang Center Hospital, Mianyang 621000, China \\ Correspondence should be addressed to Min Dai; heatedspring@sohu.com
}

Received 6 May 2020; Revised 3 August 2020; Accepted 9 November 2020; Published 21 November 2020

Academic Editor: Yuxin Wang; ywan943@163.com

Copyright ( 92020 Qingyan Qiu et al. This is an open access article distributed under the Creative Commons Attribution License, which permits unrestricted use, distribution, and reproduction in any medium, provided the original work is properly cited.

\begin{abstract}
Various diseases are spread by means of contaminated water or food, and the detection of pathogenic bacteria has great significance for securing a proper healthy environment for human beings. In this article, microfiber gratings (MFGs) were fabricated by using a high-frequency $\mathrm{CO}_{2}$ laser. The number of periods is 30 , and the length of the period is $600 \mu \mathrm{m}$. A type of biosensor is proposed in this study. Results showed that the biosensor was strongly sensitive to the concentration of Escherichia coli and a maximum sensitivity of $1.15 \mathrm{~nm} / 107 \mathrm{CFU}$ was achieved. The mechanism of real-time sensing of prepared MFG was also proposed, which could be due to relationship between dip wavelength shift and the concentration of detected bacteria. The prepared MFGs do not need any coating, and the proposed biosensor has a great potential for application in fields of medical treatment, biology, and farming.
\end{abstract}

\section{Introduction}

For several decades, detection of pathogenic bacteria has great significance in securing a proper healthy environment for human beings due to various diseases spread through contaminated water or food. Escherichia coli (E. coli) is an easy indicator for fecal coliform contamination [1]. The nonpathogenic population of $E$. coli mainly inhabits in the intestinal tract of most mammalian species including humans [2], which often causes severe intestinal and extraintestinal diseases in areas such as bloodstream, the urinary tract, and meninges [3-5]. Various techniques are developed for the detection of $E$. coli including culture methods, fluorescence, and microscopy [6]. However, most of the traditional detection methods are time-consuming and may take up to a whole week, resulting in a limitation to the extensive and real application for real-time sensing $[7,8]$. Thus, sensitive, rapid, and accurate detection methods are urgently required.

Due to their unique properties such as enhanced evanescent fields, tight light confinement, and large waveguide dispersion, microfibers have attracted extensive interests since Tong's first demonstration in 2003 [9, 10]. Microfibers and related structures were intensively investigated for various sensor applications, such as temperature sensor [11-14], RI (refractive index) refractometer [15-18], and gas sensor [19-21]. A biosensor based on conventional fused fiber coupler was proposed by Tazawa et al. in 2007 [22]. It has been known that the transmission spectrum of a microfiber is strongly affected by the RI of the surrounding medium because of evanescent field generated on the fiber surface at the fused region. Hence, a higher sensitivity can be obtained by properly decreasing the diameter of a microfiber. Microfiber structure fabricated by Liao et al. [23] as a RI sensor has a sensitivity of $2735 \mathrm{~nm} / \mathrm{RIU}$ (refractive index unit, RIU). In the article, we fabricated a type of biosensor for the detection of $E$. coli concentration based on microfiber gratings (MFGs), which has a great potential application for real-time monitoring of the growth of $E$. coli.

\section{Experimental Methods}

2.1. Microfiber Preparation. Figure 1(a) shows the microfiber fabrication diagram. Telecom single-mode fibers (SMF28, Corning) were put carefully into the taper drawing system, and then, a low-loss microfiber was fabricated by 


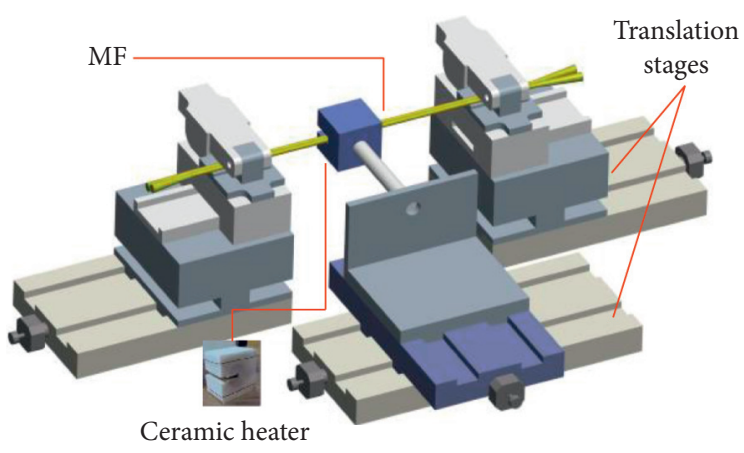

(a)

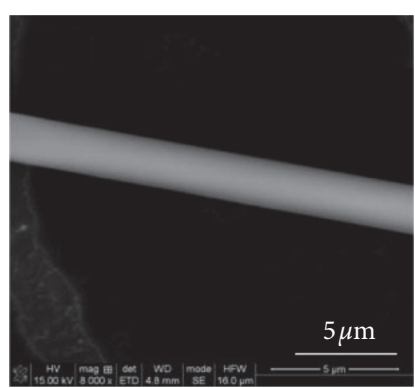

(b)

FIgURE 1: (a) Microfiber fabrication setup; (b) the SEM image of the waist region of prepared microfiber. The diameter of each fused microfiber is about $2 \mu \mathrm{m}$.

using the heating brushing technique [24]. Figure 1(b) is a scanning electron microscope (SEM) image of the waist region of the prepared microfiber, which indicates that the diameter of the microfiber is about $2 \mu \mathrm{m}$.

2.2. Sensor Fabrication. MFGs were fabricated by using a high-frequency $\mathrm{CO}_{2}$ laser. The number of periods is 30 , and the length of the period is $600 \mu \mathrm{m}$. The transmission spectrum of MFG displays resonant behavior due to periodic modulation of microfiber surface RI.

The free-standing MFG could be affected by environmental factors such as physical vibration and air flow due to its poor mechanical stability, which will produce large measurement errors and disturb sensing results.

In order to improve the mechanical stability of MFG and enhance the repeatability of the entire sensing system, packaged MFG in a surrounding structure using a low RI UV curable polymer (Thorlabs) is essential. Figure 2 shows the schematic diagram of the embedded MFG and experimental setup. A microscope slide was firstly covered with two small slides in parallel to create an open-top channel. Then, two blocks of thin slides were used to seal the two ends of the channel and to support the MFG sample in place. Then, the fabricated MFG was placed into the channel, and the entire coupler was suspended in the sensing environment. Several drops of UV curable polymer were used to fix the MFG sample. The entire sensing sample was exposed under UV radiation (UV LED system, Thorlabs) for 60 seconds. At last, an uncovered section with length about $15 \mathrm{~mm}$ in the center of the channel was formed, which was to be used for the E. coli sensing experiment. A semiconductor laser diode (SLD, Throlab S5FC 1550P-A2) with a central wavelength of $1550 \mathrm{~nm}$ was used as the optical source. It was connected to the input port of the MFG. An optical spectrum analyzer (OSA) (Yokogawa, 6370C) was connected to the outputs of the MFG to record the output transmission spectra.

2.3. Biosensing Experiment. Culture medium without bacteria is a basic element in biosensing experiment, which is significant for the culture of bacteria. Culture medium was

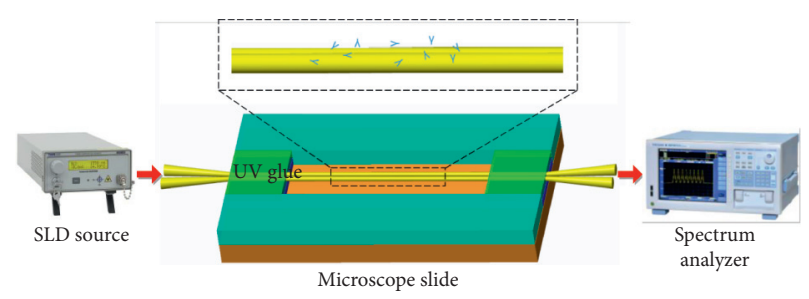

FIGURE 2: Schematic diagram of the embedded MFG and experimental setup.

preserved at $-20^{\circ} \mathrm{C}$ and refrigerated at $4^{\circ} \mathrm{C}$. Other preparation works were performed at room temperature and would last for about 5 minutes, during which the temperature of culture medium would rise. Organic contaminants adhered to the surface of the entire sensing sample, and cuvettes were removed by washing with pure ethyl alcohol.

\section{Results and Discussion}

In this experiment, temperature is a main factor for the reproduction behavior of the bacteria. Thus, the sensing process was divided into two stages. At the first stage, the culture medium had a low temperature and the reproduction of bacteria was slow. $3 \mathrm{ml}$ culture medium with an initial bacterial concentration of $1 \times 107 \mathrm{CFU} / \mathrm{ml}$ was dropped into the channel of the sensing sample. Measured spectral responses were recorded every $30 \mathrm{~min}$. At the second stage, bacteria reproduced actively at room temperature, which was much faster than that in the first stage. The initial bacterial concentration was $1.9 \times 107 \mathrm{CFU} / \mathrm{ml}$. Measured spectral responses were recorded every $5 \mathrm{~min}$. Distinguishing the two stages will contribute to the data analyzation.

For the first stage, the initial bacterial concentration was $1 \times 107 \mathrm{CFU} / \mathrm{ml}$. Measured spectral responses were recorded every $30 \mathrm{~min}$. Figure $3(\mathrm{a})$ illustrates the measured spectral responses during the first 4 hours, and Figure 3 (b) shows the dip wavelength shift corresponding to the spectral responses. The dip wavelength showed a blueshift from $1554.68 \mathrm{~nm}$ to $1550.06 \mathrm{~nm}$ with the increase of time, with an average blueshift speed of about $0.018 \mathrm{~nm} / \mathrm{min}$, which could be due 


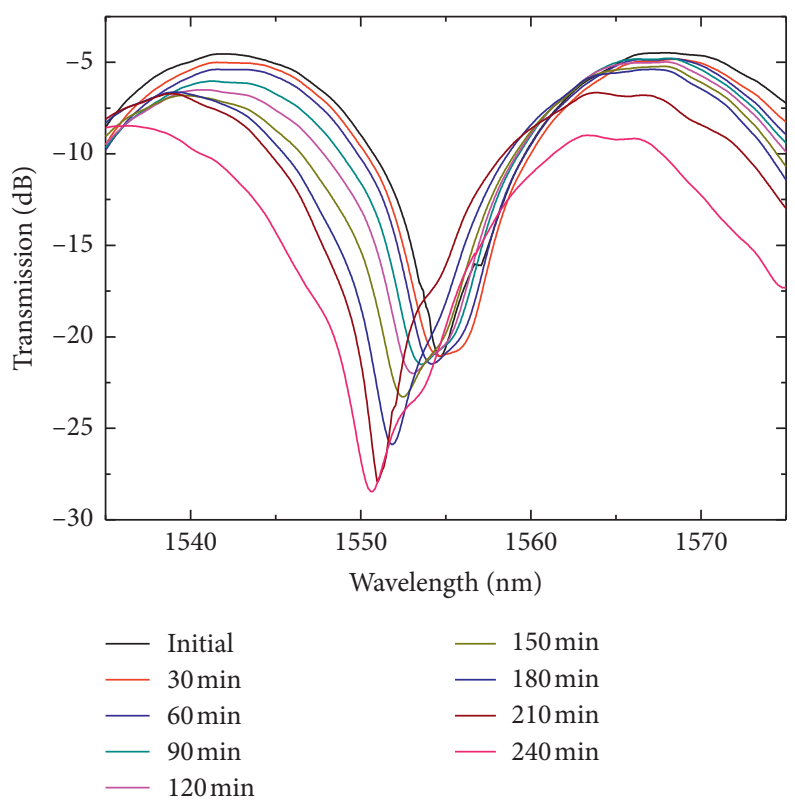

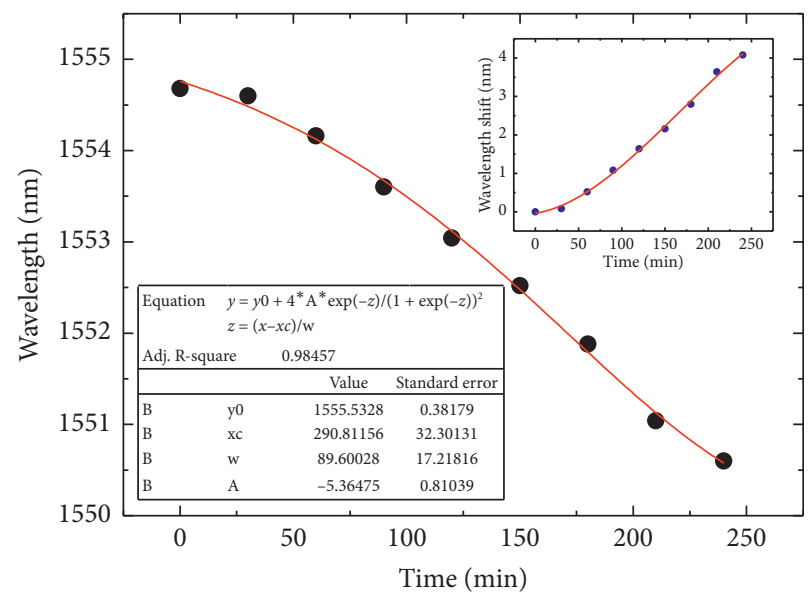

(a)

(b)

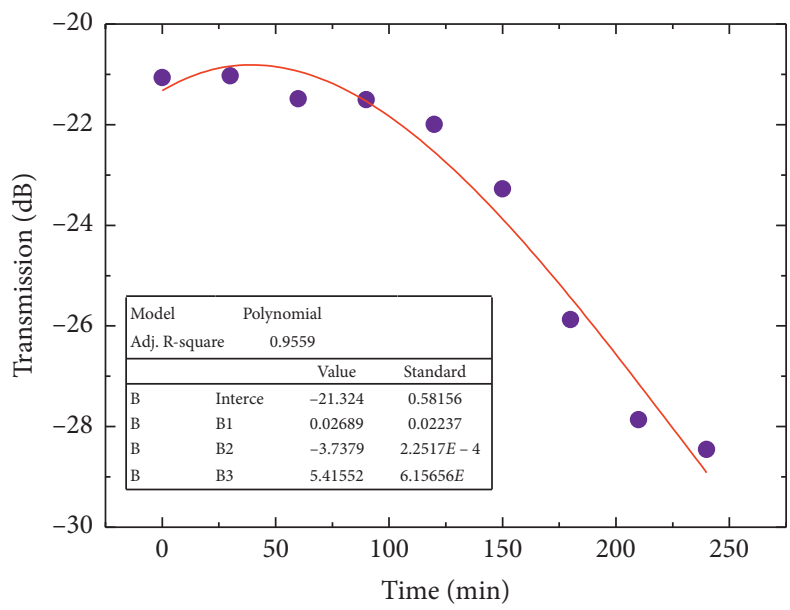

(c)

Figure 3: (a) Measured spectral responses during the first 4 hours, which was recorded every 30 min; the wavelength shift (b) and transmission loss (c) during the first 4 hours, which were caused by the change of the bacterial concentration.

to the slow reproduction of bacteria in the culture medium at low temperature. Figure 3(c) indicates the transmission varies with the increase of measuring time. The transmission increased a little bit from $-21.065 \mathrm{~dB}$ to $-21.031 \mathrm{~dB}$ during the first $30 \mathrm{~min}$, which could be due to the low concentration of bacteria in the culture medium at low temperature. The number of bacteria decreased during the first $30 \mathrm{~min}$. Then, the transmission decreased from $-21.0318 \mathrm{~dB}$ to $-28.402 \mathrm{~dB}$ with further increase of time, which could be due to the slow reproduction of $E$. coli. The number of $E$. coli gradually increased.

For the second stage, the temperature of the tested sample was the room temperature. The initial bacterial concentration was $1.9 \times 107 \mathrm{CFU} / \mathrm{ml}$. Measured spectral responses were recorded every $5 \mathrm{~min}$. Figure 4 (a) illustrates the wavelength shift corresponding to the spectral responses during the half-hour; and measured wavelength shift and transmission loss with the increase of time are given in Figures 4(b) and 4(c). Results indicate that the dip wavelength has a linear blueshift from $1550.6 \mathrm{~nm}$ to $1535.36 \mathrm{~nm}$ with the increase of time, with a blueshift speed of about $0.5 \mathrm{~nm} / \mathrm{min}$, much higher than that at the first stage $(0.018 \mathrm{~nm} / \mathrm{min})$. The fast reproduction rate of $E$. coli at the room temperature is the main reason for the fast blueshift speed at the second stage. The transmission decreased from $-28.4289 \mathrm{~dB}$ to $-38.9895 \mathrm{~dB}$. The decrease in speed of the transmission at this stage is about 12 times in comparison with that in the first stage. This phenomenon can be explained by basic biology theories that the number of bacteria in culture medium increases drastically. Experimental results indicate that both the dip wavelength and transmission can be employed to monitor the concentration 

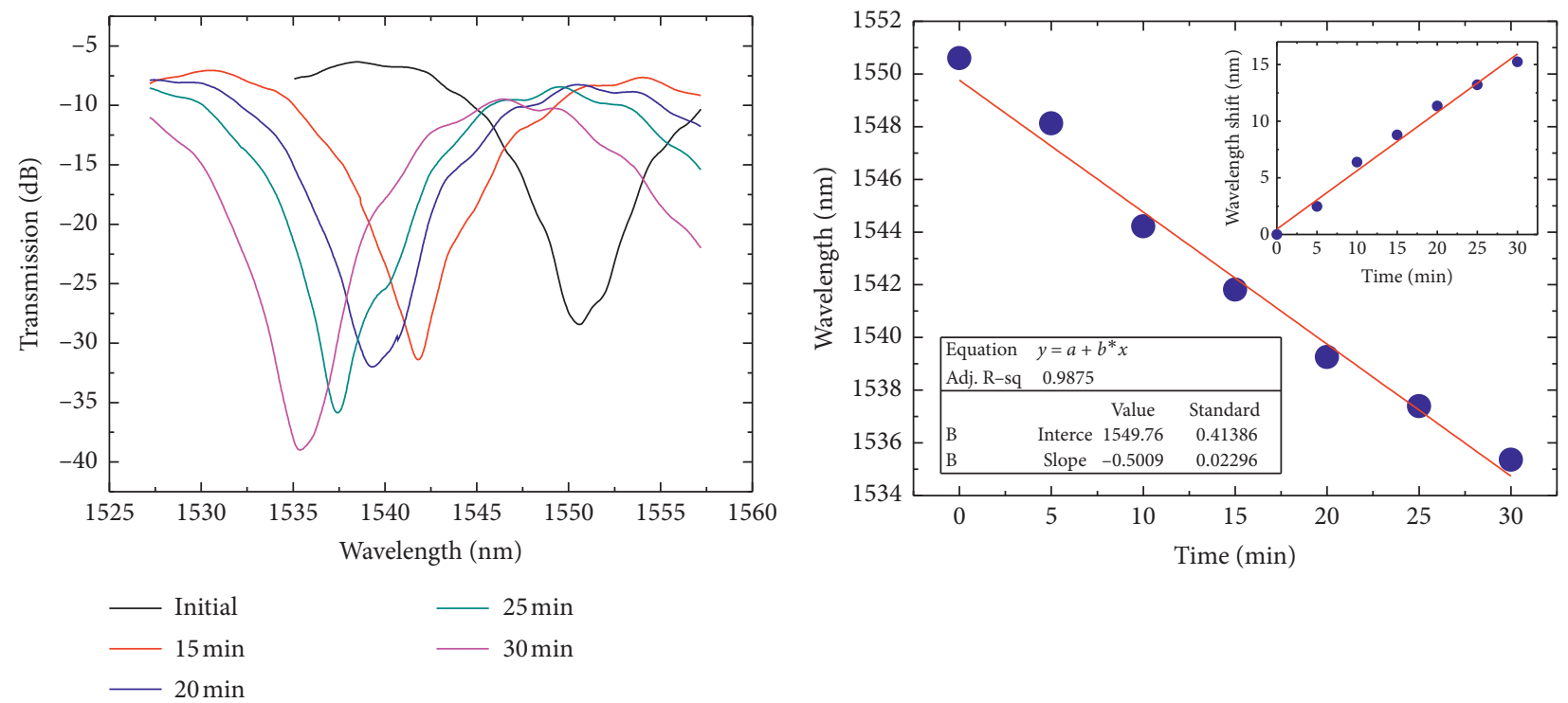

(a)

(b)

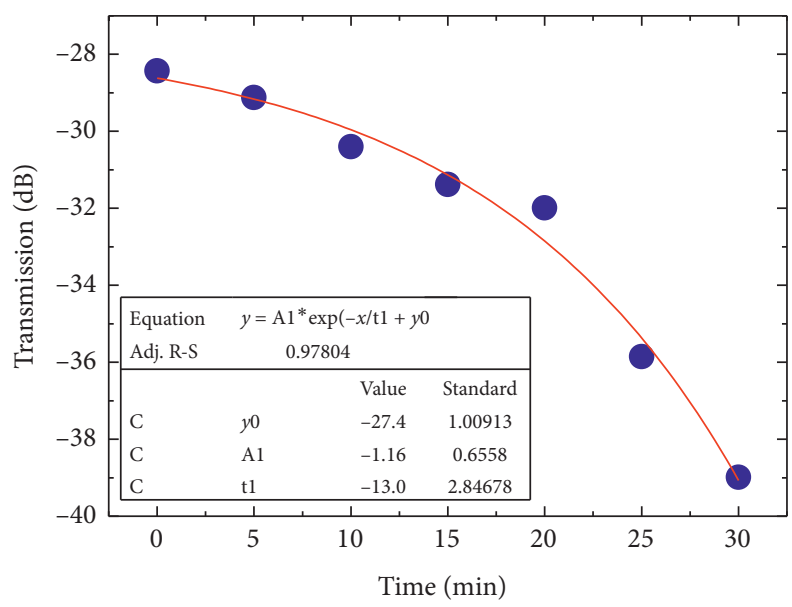

(c)

Figure 4: (a) Measured spectral responses after 4 hours, which was recorded every $5 \mathrm{~min}$; the wavelength shift (b) and transmission loss (c) caused by the change of the bacterial concentration.

of E. coli. Compared with transmission loss, dip wavelength is a more sensitive factor (2.3 times).

In order to verify the relationship between bacterial concentration and dip wavelength shift, $5 \mathrm{ml}$ culture medium with $E$. coli (the initial concentration is $0.047 \%$, $1 \times 107 \mathrm{CFU} / \mathrm{ml}$ ) was dropped into the channel of the sensing sample and cuvette, respectively. The reproduction rates of $E$. coli are the same for the culture medium with the same volume at the same temperature. OD (optical density) of the culture medium was measured by using a spectrophotometer (Shimadzu, UV-2600). The average values of OD were adopted from three cuvettes in order to obtain a higher accuracy. Spectral responses and ODs were recorded every $30 \mathrm{~min}$.

Time-dependent wavelength shift for real-time sensing is given in Figure 5(a). Resonance wavelength was recorded every $30 \mathrm{~min}$, and the total measuring time was $270 \mathrm{~min}$. The wavelength shift increased exponentially with the increase of time. At the beginning of the sensing process (within $240 \mathrm{~min}$ ), the wavelength shifts slowly due to the slow reproduction rate of $E$. coli at low temperature. While after $240 \mathrm{~min}$, the wavelength shift dramatically increased, indicating a drastic reproduction of $E$. coli at room temperature. This result is consistent with result in Figures 3 and 4 . Figure 5(b) illustrates the exponential relationship between OD and dip wavelength. The dip wavelength shows a blueshift from $1554.68 \mathrm{~nm}$ to $1515.26 \mathrm{~nm}$ with the increase of OD. OD is proportional to the concentration of E. coli in culture medium. It indicates that the concentration of $E$. coli can be obtained from the value of wavelength shift according to expressions in Figure 5.

The behavior of the prepared sensor for the detection of the concentration of E. coli is shown in Figure 6. It indicates that the detected signal increased monotonously with the increasing concentration of $E$. coli. An exponent fitting was applied to the sensitivity variation with respect to dip 


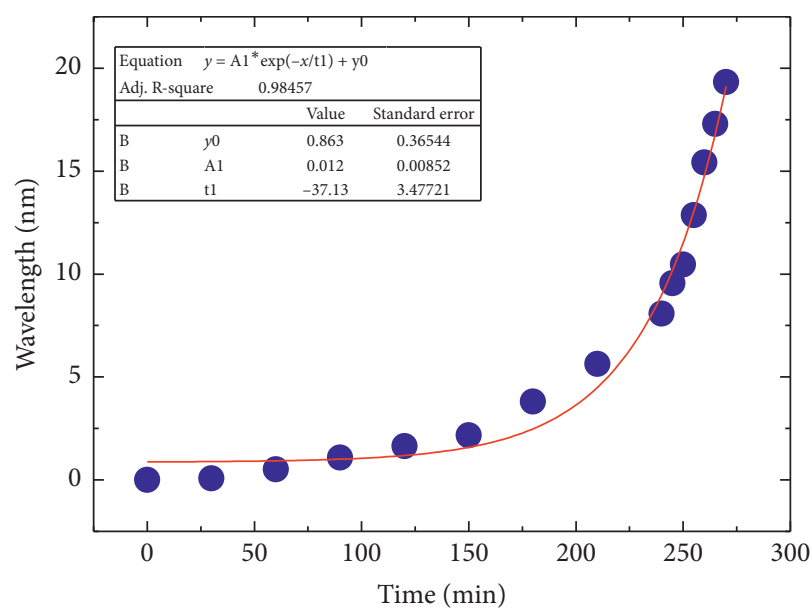

(a)

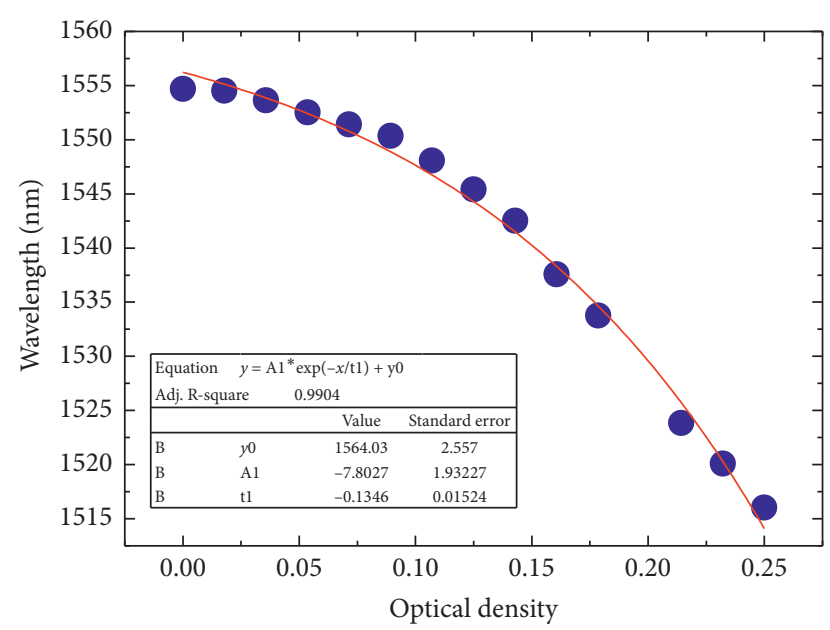

(b)

FIGURE 5: (a) Time-dependent wavelength shift and resonance wavelength was recorded every $30 \mathrm{~min}$. (b) The relationship between dip wavelength and optical density.

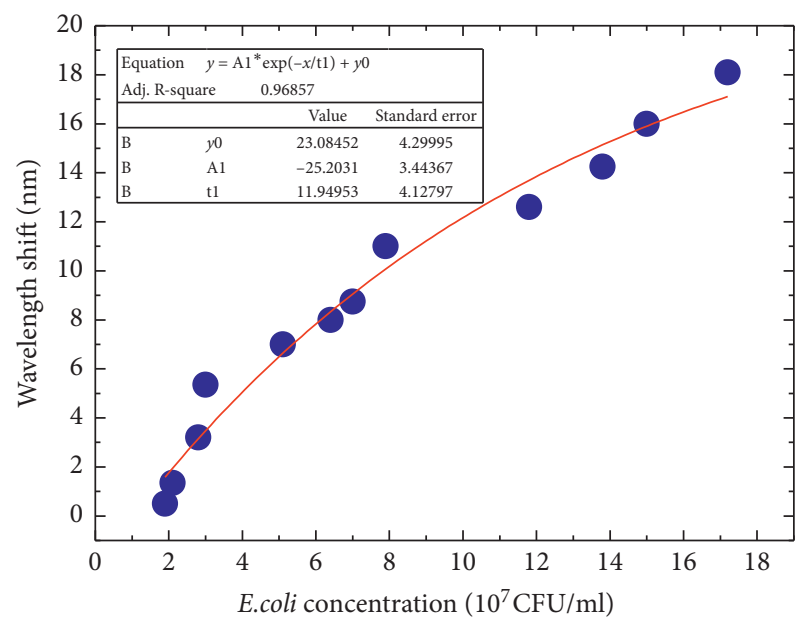

FIgURE 6: The behavior of prepared sensor for the detection of the E. coli. Experimental results were recorded after the culture medium was exposed at room temperature $1 \mathrm{~h}$ later.

wavelength shift in Figure 6. A maximum sensitivity of $1.15 \mathrm{~nm} / 107 \mathrm{CFU}$ was achieved by using the fabricated sensor. It implies that real-time sensing of concentration of E. coli can be achieved by using the MFG sample according to the relationship between dip wavelength shift and the concentration of detected bacteria.

Generally, the MFGs can allow power transfer between the guided modes when a certain resonant condition is satisfied, which leads to a series of transmission dips in the spectrum. The coupling of modes with high diffraction orders has been first demonstrated in conventional fibers. The MFGs can be used in simultaneous sensing application. The resonant condition of the MFGs can be expressed as

$$
\lambda_{\text {res }}=\frac{\left(n_{\text {eff } 1}-n_{\text {eff } 2}\right) \Lambda}{N},
$$

where $\lambda_{\text {res }}$ is the resonant wavelength, $\Lambda$ is the grating period, $N$ is the diffraction order, and $n_{\text {eff1 }}$ and $n_{\text {eff2 }}$ are the effective indices for the lower- and higher-order guided modes, respectively. Based on Eq. (1), for the same resonant wavelength and the same coupled modes, the larger value of $N$ has the longer grating period $\Lambda$. The variation of ambient refractive index (nex) can produce different changes to the dissimilar mode indices, which leads to a modification of the mode-index difference and induces the shift of the spectrum. By taking a small variation of $n_{\text {ex }}$ from Eq. (1), the sensitivity of dip wavelength to refractive index can be expressed as

$$
S=\frac{\mathrm{d} \lambda}{\mathrm{d} n}=\frac{\lambda \partial \Delta n / \partial n_{\mathrm{ex}}}{(\Delta n-\lambda \partial \Delta n) / \partial \lambda} .
$$

It is shown that the sensitivity is independent on either the diffraction order or the grating period, but it is dependent on the microfiber diameter and the operating wavelength.

\section{Conclusion}

Real-time sensing for the concentration of $E$. coli based on MFG structure is proposed and experimentally demonstrated in this article. The MFG sample was fabricated based on the taper drawing system by using the heating brushing technique. The performance of the sensor in culture medium with $E$. coli in the wavelength domain was evaluated. Results showed that compared with transmission loss, dip wavelength is more sensitive to the concentration of bacteria. The MFG-based sensor is capable of real-time sensing for the concentration of $E$. coli. A maximum sensitivity of $1.15 \mathrm{~nm} /$ $107 \mathrm{CFU}$ was achieved in our experiment. The detection mechanism could be due to the relationship between dip wavelength shift and the concentration of bacteria. The fabricated MFG structure does not need any coating. Considering the simple structure, compact size, low cost, and high sensitivity of the proposed MFG-based sensor, this 
research offers a sensitive, rapid, and accurate solution for the detection of bacteria in advanced biology fields.

\section{Data Availability}

Data used to support the findings of this study are included within the article.

\section{Conflicts of Interest}

The authors declare that there are no conflicts of interest regarding the publication of this paper.

\section{Acknowledgments}

The authors thank Laser Fusion Research Center for providing the samples. This work was supported by the National Natural Science Foundation of China (NSFC) (Grant No. 61705205).

\section{References}

[1] S. Bonacorsi and E. Bingen, "Molecular epidemiology of Escherichia coli causing neonatal meningitis," International Journal of Medical Microbiology, vol. 295, no. 6-7, pp. 373381, 2005.

[2] C. F. Marrs, L. Zhang, and B. Foxman, "Escherichia colimediated urinary tract infections: are there distinct uropathogenic E. coli (UPEC) pathotypes?" Fems Microbiology Letters, vol. 252, no. 2, pp. 183-190, 2005.

[3] Y. H. Hui, R. Gorham, K. D. Murrell et al., Foodborne Disease Handbook, Marcel Dekker, New York City, NY, USA, 1994.

[4] E. J. Klein, D. R. Boster, J. R. Stapp et al., "Diarrhea etiology in a children's hospital emergency department: a prospective cohort study," Clinical Infectious Diseases, vol. 43, no. 7, pp. 807-813, 2006.

[5] J. R. Johnson, "Microbial virulence determinants and the pathogenesis of urinary tract infection," Infectious Disease Clinics of North America, vol. 17, no. 2, pp. 261-278, 2003.

[6] A. K. Bej, M. H. Mahbubani, J. L. Dicesare, and R. M. Atlas, "Polymerase chain reaction-gene probe detection of microorganisms by using filter-concentrated samples," Applied and Environmental Microbiology, vol. 57, no. 12, pp. 3529-3534, 1991.

[7] M. Kandpal, R. K. Gundampati, and M. Debnath, "Surface charge based rapid method for detection of microbial contamination in drinking water and food products," World Academy of Science Engineering \& Technology, vol. 58, 2009.

[8] V. Dixit, J. C. Tewari, and B. S. Sharma, "Detection of E. coli in water using semi-conducting polymeric thin film sensor," Sensors and Actuators B: Chemical, vol. 120, no. 1, pp. 96-103, 2006.

[9] L. Tong, R. R. Gattass, J. B. Ashcom et al., "Subwavelengthdiameter silica wires for low-loss optical wave guiding," Nature, vol. 426, no. 6968, pp. 816-819, 2004.

[10] L. Tong, J. Lou, and E. Mazur, "Single-mode guiding properties of subwavelength-diameter silica and silicon wire waveguides," Optics Express, vol. 12, no. 6, pp. 1025-1035, 2004.

[11] J.-1. Kou, J. Feng, L. Ye, F. Xu, and Y.-Q. Lu, "Miniaturized fiber taper reflective interferometer for high temperature measurement," Optics Express, vol. 18, no. 13, pp. 14245-14250, 2010.
[12] H. Yang, S. Wang, X. Wang, J. Wang, and Y. Liao, "Temperature sensing in seawater based on microfiber knot resonator," Sensors, vol. 14, no. 10, pp. 18515-18525, 2014.

[13] I. Hernandez-Romano, D. Monzon-Hernandez, C. MorenoHernandez, D. Moreno-Hernandez, and J. Villatoro, "Highly sensitive temperature sensor based on a polymer-coated microfiber interferometer," IEEE Photonics Technology Letters, vol. 27, no. 24, pp. 2591-2594, 2015.

[14] P. Wang, M. Ding, G. Bramilla et al., "High temperature performance of an optical microfibre coupler and its potential use as a sensor," Electronics Letters, vol. 5, no. 48, pp. 283-284, 2012.

[15] J. Wo, G. Wang, Y. Cui et al., "Refractive index sensor using microfiber-based Mach-Zehnder interferometer," Optics Letters, vol. 37, no. 1, pp. 67-69, 2012.

[16] W. B. Ji, H. H. Liu, S. C. Tjin, K. K. Chow, and A. Lim, "Ultrahigh sensitivity refractive index sensor based on optical microfiber," IEEE Photonics Technology Letters, vol. 24, no. 20, pp. 1872-1874, 2012.

[17] Z. Xu, Q. Sun, B. Li et al., "Highly sensitive refractive index sensor based on cascaded microfiber knots with vernier effect," Optics Express, vol. 23, no. 5, pp. 6662-6672, 2015.

[18] L. Bo, P. Wang, and Y. Semenova, G. Farell, High sensitivity fiber refractometer based on an optical microfiber coupler," IEEE Photonics Technology Letters, vol. 25, no. 3, pp. 228-230, 2013.

[19] Y. Wu, B. Yao, A. Zhang et al., "Graphene-coated microfiber Bragg grating for high-sensitivity gas sensing," Optics Letters, vol. 39, no. 5, pp. 1235-1237, 2014.

[20] B. Yao, Y. Wu, Y. Cheng et al., "All-optical Mach-Zehnder interferometric $\mathrm{NH}_{3}$ gas sensor based on graphene/microfiber hybrid waveguide," Sensors and Actuators B: Chemical, vol. 194, pp. 142-148, 2014.

[21] C.-B. Yu, Y. Wu, X.-L. Liu et al., "Graphene oxide deposited microfiber knot resonator for gas sensing," Optical Materials Express, vol. 6, no. 3, pp. 727-733, 2016.

[22] H. Tazawa, T. Kanie, and M. Katayama, "Fiber-optic coupler based refractive index sensor and its application to biosensing," Applied Physics Letters, vol. 91, no. 11, p. 677, 2007.

[23] C. R. Liao, D. N. Wang, X. He, and M. W. Yang, "Twisted optical microfibers for refractive index sensing," IEEE Photonics Technology Letters, vol. 23, no. 13, pp. 848-850, 2011.

[24] G. Brambilla, V. Finazzi, and D. J. Richardson, "Ultra-low-loss optical fiber nanotapers," Optics Express, vol. 12, no. 10, pp. 2258-2263, 2004. 